\title{
The impact of hysteresis on the electrocaloric effect at first-order phase transitions
}

\author{
Madhura Marathe, ${ }^{1}$ Claude Ederer, ${ }^{1}$ and Anna Grünebohm $\left.{ }^{2}, \mathrm{a}\right)$ \\ ${ }^{1)}$ Materials Theory, ETH Zürich, Wolfgang-Pauli-Str. 27, 8093 Zürich, Switzerland \\ ${ }^{2)}$ Faculty of Physics and CENIDE, University of Duisburg-Essen, 47048 Duisburg, \\ Germany
}

\begin{abstract}
We study the impact of thermal hysteresis at the first-order structural/ferroelectric phase transitions on the electrocaloric response in bulk $\mathrm{BaTiO}_{3}$ by performing molecular dynamics simulations for a first-principlesbased effective Hamiltonian. We demonstrate that the electrocaloric response can conceptually be separated in two contributions: a transitional part, stemming from the discontinuous jump in entropy at the first order phase transition, and a configurational part, due to the continuous change of polarization and entropy within each phase. This latter part increases with the strength of the applied field, but for small fields it is very small. In contrast, we find a large temperature change of $\sim 1 \mathrm{~K}$ resulting from the transition entropy, which is essentially independent of the field strength. However, due to the coexistence region close to the first order phase transition, this large electrocaloric response depends on the thermal history of the sample and is generally not reversible. We show that this irreversibility can be overcome by using larger fields.
\end{abstract}

Keywords: Electrocaloric effect, molecular dynamics simulations, hysteresis, first-order phase transitions, $\mathrm{BaTiO}_{3}$.

\section{INTRODUCTION}

The electrocaloric (EC) effect results in an adiabatic temperature change (or an isothermal entropy change) with variation of an external electric field ${ }^{112}$ Along with the magnetocaloric and elastocaloric effects, the EC effect has the potential to lead to energy-efficient and environmentally friendly solid-state cooling devices $\frac{3 \sqrt{4}}{\text { Typ- }}$ ically, the largest caloric response is observed near ferroic phase transitions. ${ }^{3}$ In particular, giant temperature changes have been found at first-order (FO) phase transitions with coupled ferroic and structural degrees of freedom. tence region exists, which leads to thermal hysteresis, i.e., within a certain temperature range, the system can exist in different (meta-)stable states depending on the history of the sample. Thus, at the same temperature and applied field strength, the state of the system can differ in field cooled (FC), field heated $(\mathrm{FH})$, zero-field cooled (ZFC), or zero-field heated (ZFH) samples.

The impact of thermal hysteresis on the reversibility of the caloric response has been studied extensively for the case of magnetic materials close to magneto-structural phase transitions ${ }^{4}-7$ It has been shown that for systems with broad hysteresis, a giant caloric response can typically only be found for the first field pulse. However, for cooling applications, a device needs to be operated over a large number of field cycles. Therefore, the search for hysteresis-free materials, ways to bypass the thermal hysteresis, or the use of smaller reversible responses within the coexistence region is a very active area in the field of magnetic materials ${ }^{[5]}$ In contrast, there are only few such studies for the EC effect. Thus, a better under-

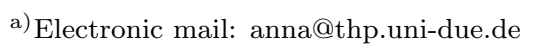

standing of the impact of thermal hysteresis is needed for future applications of EC materials.

The EC effect has been extensively studied in $\mathrm{BaTiO}_{3}$ (BTO), see, e.g., 8 17, BTO exhibits a paraelectric (PE) cubic (C) phase at high temperatures and, on cooling, undergoes three transitions to ferroelectric (FE) tetragonal $(\mathrm{T})$, orthorhombic $(\mathrm{O})$, and rhombohedral $(\mathrm{R})$ phases. In absence of an external field, each $\mathrm{FE}$ transition in BTO is a FO transition. ${ }^{18}$ For small fields, this results in thermal hysteresis and coexistence regions, in which the state of the system depends on the preceding heat treatment. Previous studies have shown that under increasing strength of an applied field, the transition temperatures are shifted, the thermal hysteresis is reduced, and for sufficiently large fields the nature of the transition can change 19 21 For the PE-FE transition, there is no welldefined phase transition any more above a critical field strength $E_{\mathrm{c}}$. 10

Some experimental measurements performed at small applied fields have indeed reported irreversibility in the EC response of BTO. For example, Moya, et al ${ }^{9}$ have found an irreversible EC response at the PE-FE transition. Furthermore, around the FE-FE (T-O) transition, Bai, et al.$^{[22}$ measured a large EC response only during the first application of an electric field, which was then reduced to a rather small response on further cycling, analogous to the irreversibility found at magneto-structural phase transitions 67

Most theoretical studies so far have focused on the EC effect close to the PE-FE transition under large fields. Since at large fields $\left(E>E_{\mathrm{c}}\right)$ there is no FO phase transition, 10/21 irreversible effects are not expected in this case. On the other hand, an irreversible EC effect has been predicted even for large fields, when different metastable states occur in defect-doped BTO 16 Under small fields, thermal hysteresis exists at all transitions, and thus an irreversible EC effect is expected, which de- 


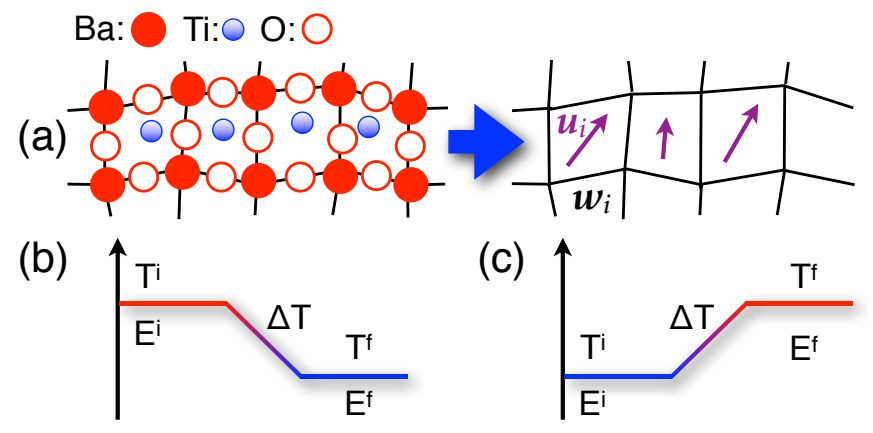

FIG. 1. (a) Illustration of the construction of the effective Hamiltonian: the local atomic arrangement is mapped on the local soft mode vectors $\mathbf{u}_{\mathbf{i}}$ and the local strains $\mathbf{w}_{\mathbf{i}}$ in each unit cell. (b)-(c) Illustration of the direct simulation of the EC temperature change: the system is equilibrated in an external field $E_{i}$ at the start temperature $T_{i}$, the field is ramped up or down to the final field $E_{f}$ and after equilibrium is reached, the final temperature $T_{f}$ is recorded. $\Delta T$ indicates the adiabatic temperature change on varying the field. For normal EC response, $\Delta T$ is negative when the field is ramped down (b) and positive when the field is ramped up (c).

pends strongly on the thermal history of the sample.

In this paper, we study the EC response of BTO at applied fields that are smaller than the critical fields. In particular, we examine how the EC effect depends on the thermal history of the sample, and whether periodic cycling of the field near the coexistence region results in irreversibilities. To this end, we perform molecular dynamics (MD) simulations for an effective Hamiltonian derived from first principles (see, e.g., Ref. 17). We focus on the EC response around the $\mathrm{C}-\mathrm{T}$ and $\mathrm{T}-\mathrm{O}$ transitions. We first show that, within the coexistence region, the EC temperature change indeed depends on the initial phase of the system. Then, we study the EC response for different applied field strengths around the critical field while switching the field on and off. Further, we apply a field for up to two cycles at a few selected temperatures to examine the reversibility of the response. We observe that the effect is irreversible for small fields, with a large response in the first cycle, which then reduces by an order of magnitude in the following cycle. Finally, we briefly compare the observed behavior to the well-known hysteretic effects in materials showing a FO magnetostructural phase transition.

\section{COMPUTATIONAL DETAILS}

To construct the effective Hamiltonian used in our work, the atomic degrees of freedom in each unit cell $i$ are mapped on a ferroelectric soft mode vector $\left(\mathbf{u}_{i}\right)$ and

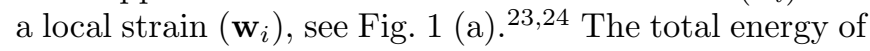
the system is then expressed as a low order polynomial in terms of these variables. All parameters of this effective Hamiltonian have been calculated using $a b$ initio density functional theory ${ }^{25}$ We use the open-source feram code (http://loto sourceforge.net/feram/) ${ }^{26}$, which allows to perform MD simulations for the effective Hamiltonian at finite temperatures and fields. We use a $96 \times 96 \times 96 \mathrm{su}-$ percell to simulate bulk BTO within periodic boundary conditions. For computational efficiency, we treat only the soft mode variables as dynamical variables whereas the local and global strains are optimized in each MD step according to the current soft mode configuration.

This method has been successfully used to calculate the EC response, see e.g., 13 17, and 27. We calculate the response in bulk BTO using the direct method. First, we allow the system to equilibrate at a given initial temperature $T_{\mathrm{i}}$ and initial field $E_{\mathrm{i}}$ using sufficiently long thermalization times in the MD simulations. Then, the applied electric field is either switched off (ramp down) or on (ramp up), as illustrated in Figs. 11(b) and (c) respectively. In this study, we always apply the field along the pseudo-cubic [001] direction. During and after the fieldramping, we allow the system to evolve and then equilibrate again within the constant energy (microcanonical) ensemble before averaging the physical quantities of interest. The corresponding EC temperature change $\Delta T$ is obtained as difference between the initial and final temperatures. Note that the effective Hamiltonian results in a reduction of degrees of freedom from 15 in the real system to 3 in the model system. Therefore, the change in temperature is rescaled by a factor of $1 / 5$. More details of our calculations are described in Refs. 17 and 21 .

To study the impact of thermal hysteresis, i.e., the thermal history of the sample, we prepare the system by either heating or cooling simulations under a constant external field. At each temperature $T$, local dipole configurations are obtained after thermalization and then used as initial configuration to calculate the EC temperature change. In addition, we have also performed simulations where the starting configuration was obtained by simply randomly initializing the local dipoles and subsequent thermalization at a given temperature and field, ensuring that the system is in a well-defined initial state, before ramping the field either up or down from a given initial temperature and monitoring the asymmetry of the obtained EC temperature change.

\section{RESULTS AND DISCUSSION}

First, we investigate the influence of the thermal history on the EC effect of BTO. We calculate the EC response for ramping down a field of $E_{\mathrm{i}}=20 \mathrm{kV} / \mathrm{cm}$ applied along [001] near the C-T and T-O transitions. This field strength is below the critical field strength found for our model and thus there is thermal hysteresis at both transitions. ${ }^{21}$ To determine the influence of the thermal history, we perform two simulations for each $T_{\mathrm{i}}$ using the two starting configurations obtained from $\mathrm{FH}$ and $\mathrm{FC}$ simulations.

The calculated $\Delta T$ as function of initial temperature is 


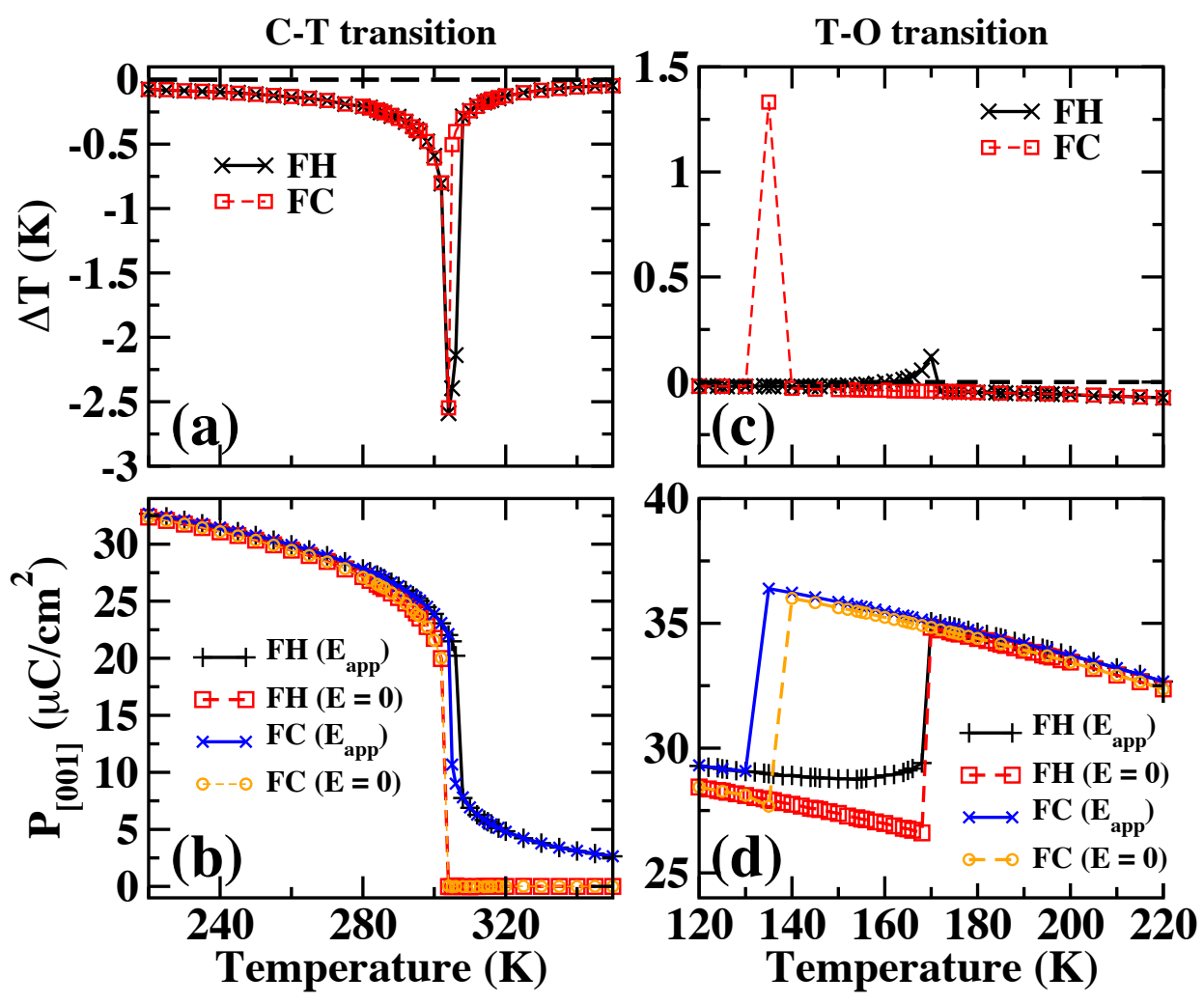

FIG. 2. The EC temperature change is plotted as a function of initial temperature for an applied field of $20 \mathrm{kV} / \mathrm{cm}$ around the $\mathrm{C}-\mathrm{T}$ transition (a) and the T-O transition (c). Two differently prepared initial configurations (FC and FH) are used at each temperature, leading to different EC response for the same applied field. The corresponding polarization components along the applied field direction, $P_{[001]}$, are plotted in (b) and (d) before (solid lines) and after (dashed lines) the field has been removed.

plotted in Fig. 2(a) and (c). We observe a clear difference between the EC response calculated for the $\mathrm{FH}$ and $\mathrm{FC}$ starting configurations. At the PE-FE (C-T) transition, we obtain a peak value of $|\Delta T| \sim 2.5 \mathrm{~K}$ at $304 \mathrm{~K}$ for both $\mathrm{FC}$ and $\mathrm{FH}$ starting configurations. However, the peak is sharper (only one data point) for the FC configurations as compared to the $\mathrm{FH}$ case, for which the peak extends up to $T_{\mathrm{i}}=306 \mathrm{~K}$. At the T-O transition, we obtain a sharp peak at $135 \mathrm{~K}$ for the FC case with $|\Delta T| \sim 1.3 \mathrm{~K}$. The response is inverse, i.e., positive $\Delta T$ under field removal, as expected for this transition and field along [001], see Ref. 21. For the FH case we observe only a small feature around $170 \mathrm{~K}$ with a magnitude of $\sim 0.12 \mathrm{~K}$ and a broad decrease towards the low- $\mathrm{T}$ side.

To better understand these differences between the FC and FH response, Figs. 2(b) and (d) show the components of the electric polarization along the applied field direction as function of initial temperature for the initial and final states, respectively, i.e., with and without applied field. One recognizes that the difference in the $\mathrm{FC}$ and $\mathrm{FH} \mathrm{EC}$ response is related to differences in the initial and/or final states of the system. At the PE-FE transition, the $\mathrm{FH}$ initial configurations are in the FE-T phase up to $T_{\mathrm{i}}=306 \mathrm{~K}$, whereas in the $\mathrm{FC}$ case, the system is initially in the FE-T phase only for $T_{\mathrm{i}} \leq 304 \mathrm{~K}$. Consequently, the corresponding EC response differs for $304 \mathrm{~K}<T_{\mathrm{i}} \leq 306 \mathrm{~K}$. The final states after field removal are the same in both cases, for $T_{\mathrm{i}}<304 \mathrm{~K}$ the system ends up in the FE phase, whereas for $T_{\mathrm{i}} \geq 304 \mathrm{~K}$ it ends up in the PE phase with zero polarization. Thus, one also recognizes that the large EC response with $|\Delta T|>2 \mathrm{~K}$ occurs only in those cases where the system undergoes a transition from the FE to the PE phase under field removal. In all other cases the removal of the electric field only leads to a reduction or vanishing of (induced) polarization, but the system does not undergo a phase transition. In these cases the EC response is much smaller $(|\Delta T| \leq 1 \mathrm{~K})$.

Conceptually, the EC response can therefore be divided into two different contributions - (i) a configurational part, resulting from continuous changes of polarization and entropy induced by the applied field without triggering a phase transition, and (ii) a transitional part related to the discontinuous jump of entropy related to the field-induced FO phase transition. The analysis of Fig. 2(a) and (b) shows that for fields of the order of 
$20 \mathrm{kV} / \mathrm{cm}$ the large EC response of BTO can mainly be attributed to the transitional part (ii), whereas the configurational part (i) is very small.

The same holds true at the T-O transition. For the FC simulations, the large peak in $\Delta T$ occurs at $T_{\mathrm{i}}=134 \mathrm{~K}$, i.e., on the low temperature side of the coexistence region, when the system can undergo a phase transition from $\mathrm{T}$ to $\mathrm{O}$ under field removal. This can be seen from the polarization components in the initial and final states shown in Fig. 2(d). At all other temperatures, no phase transition occurs, and the EC response is negligibly small. For the FH starting configurations, the system is initially in the $\mathrm{O}$ phase up to the (field-dependent) high- $\mathrm{T}$ side of the coexistence region. Since an applied field of $20 \mathrm{kV} / \mathrm{cm}$ is not sufficient to shift this phase boundary below the ZFC transition temperature, $\stackrel{21}{2}$ the system remains in the O phase under field removal. Thus, no phase transition occurs and the EC response remains small over the whole temperature region around the $\mathrm{T}-\mathrm{O}$ transition. The small feature in $\Delta T$ below $170 \mathrm{~K}$, i.e., just below the FH T-O transition, is due to an enhanced polarization induced by the applied field. We note that the field-dependent phase transition lines are rather steep (see Ref. 21) and thus the temperature ranges where a field-induced transition can occur under small fields are extremely narrow. Thus, whether we obtain one or more data points with a phase transition strongly depends on the $T$-sampling.

Up to now, we have examined how the thermal hysteresis affects the EC response at both $\mathrm{C}-\mathrm{T}$ and $\mathrm{T}-\mathrm{O}$ transitions. Next, we focus on the PE-FE (C-T) transition and compare the EC effect under field application and removal, respectively, thereby also considering the impact of the strength of the applied field. In this case, we use starting configurations which have been obtained after random initialization of the local dipoles and subsequent thermalization at the initial temperature $T_{\mathrm{i}}$, either with or without applied field. Fig. 3 shows the resulting EC temperature change as function of $T_{\mathrm{i}}$ for several applied field strengths. The data-sets with positive $\Delta T$ correspond to application of the field ("on"), whereas the data-sets with negative $\Delta T$ correspond to removal of the field ("off"). Two main features can be recognized: i) for small fields $\left(E_{\text {app }}<50 \mathrm{kV} / \mathrm{cm}\right)$ the magnitude and shape of the peaks in the EC effect differ significantly between the on and off cases, and ii) for larger fields $\left(E_{\text {app }}=50 \mathrm{kV} / \mathrm{cm}\right)$ the $\Delta T$ curves look similar for the on/off cases but are shifted against each other on the temperature axis. This latter effect can easily be understood from the condition for a completely reversible EC cycle (see Ref. 17):

$$
\Delta T_{\text {off }}\left(T_{\mathrm{i}}+\Delta T_{\text {on }}\left(T_{\mathrm{i}}\right)\right)=-\Delta T_{\text {on }}\left(T_{\mathrm{i}}\right) .
$$

Here, $\Delta T_{\text {off }}$ and $\Delta T_{\text {on }}$ correspond to the EC temperature changes under field removal and application, respectively. Note, however, that Eq. (1) is not directly applicable to the data shown in Fig. 3(a), since $\Delta T$ has been corrected according to the reduced number of degrees of freedom within the effective Hamiltonian (see Sec. II). Therefore,

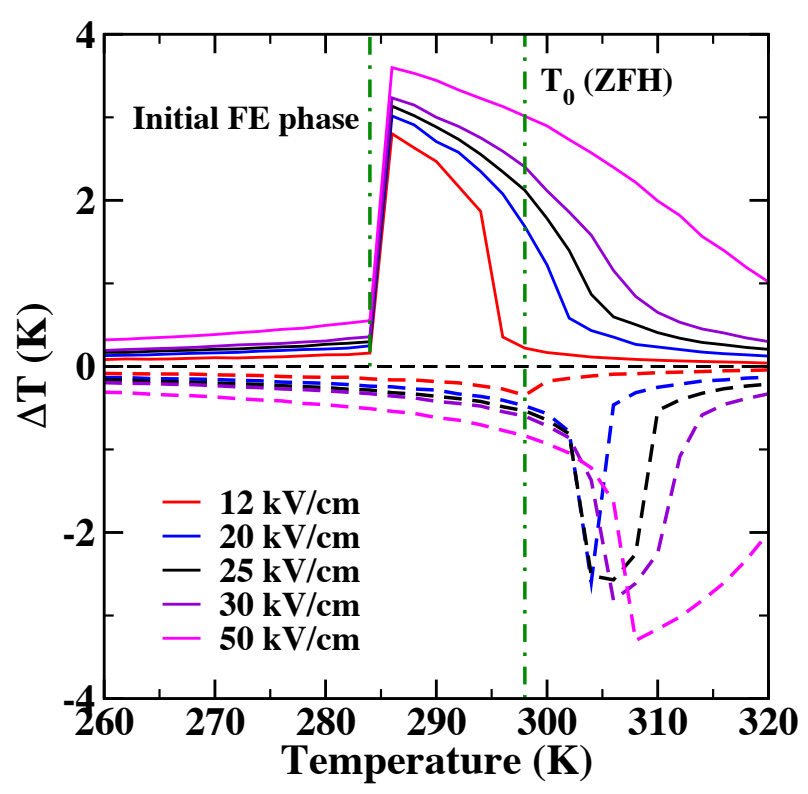

FIG. 3. EC temperature change as function of the initial temperature $T_{\mathrm{i}}$ corresponding to application (solid lines) and removal (dashed lines) of the field for different applied field strengths. In these simulations, the on/off cases are calculated independently based on randomly initialized starting configurations. The $\mathrm{ZFH}$ transition temperature $T_{0}(\mathrm{ZFH})$ is indicated by the vertical dot-dashed line. The other vertical dot-dashed line at $T=284 \mathrm{~K}$ indicates the temperature where the initial state for the "on" case changes from FE at lower temperatures to $\mathrm{PE}$ at higher temperatures.

the shift in the peak positions in Fig. 33(a) appears to be much larger than the magnitude of $\Delta T$.

To better understand the difference between the on and off cases for small applied fields, we also analyze the field-induced change in polarization, $\Delta P$ :

$$
\Delta P=P_{f}\left(T_{f}, E_{f}\right)-P_{i}\left(T_{i}, E_{i}\right) \quad .
$$

Here, $P_{i}$ and $P_{f}$ denote the polarization in the initial and final states, i.e., before and after the field ramping. Fig. 4 (a) and (b) depict $\Delta P$ as function of applied field for a few selected temperatures around the peak in $\Delta T$ for the on and off cases, respectively. We first note that for the on case, our initialization (with $E_{\mathrm{i}}=0$ ) has put the system in the $\mathrm{FE}$ phase for all temperatures below $T_{\mathrm{i}}=284 \mathrm{~K}$ and in the PE state for all temperatures above that. Thus, below $284 \mathrm{~K}$, application of an electric field leads only to minute changes in polarization (see, e.g., data for $T=280 \mathrm{~K}$ in Fig. $4(\mathrm{a})$ ) and consequently the EC effect is very small at this temperature. At slightly higher temperatures, e.g., $T=288 \mathrm{~K}$, even a small field of $12 \mathrm{kV} / \mathrm{cm}$ can trigger the FO phase transition to the FE state, leading to a steep rise in $\Delta T$ due to the large transitional contribution to the EC effect. ${ }^{28}$ Further increasing the field strength at this temperature results only in a small further increase of $\Delta T$, due to an increased configurational contribution. At temperatures close to the $\mathrm{ZFH}$ 


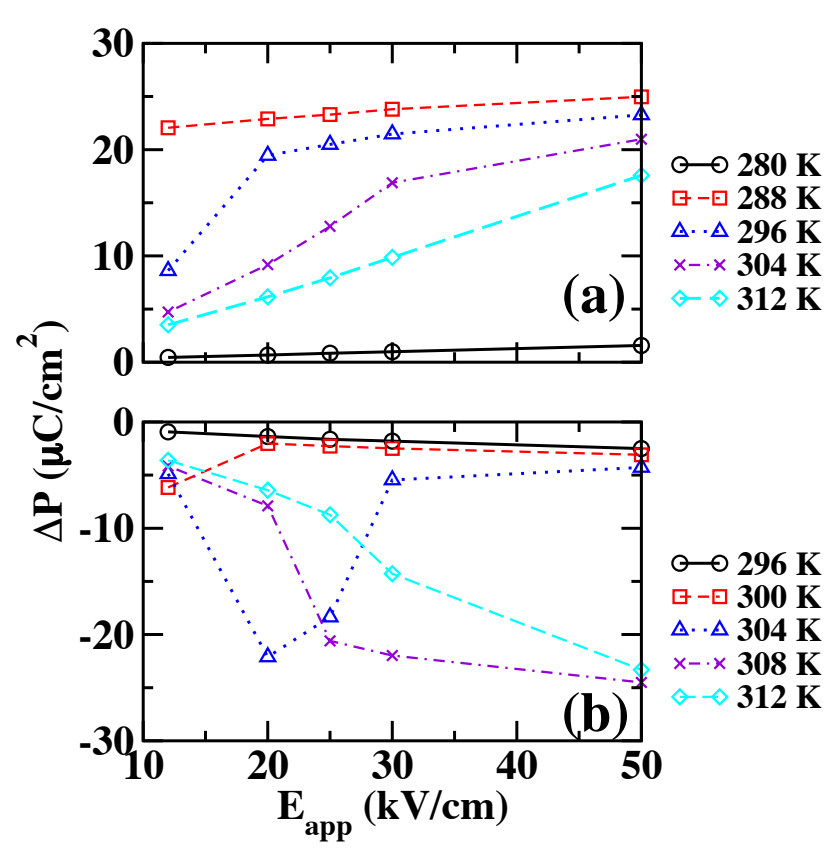

FIG. 4. Change in polarization, $\Delta P$, between final and initial states for the on (a) and off (b) ramping of the field for different applied field strengths at a few selected temperatures corresponding to the caloric response in Fig. 3.

transition temperature, e.g., $T=296 \mathrm{~K}$, a small field of $12 \mathrm{kV} / \mathrm{cm}$ is not sufficient to trigger the transition to the FE phase (note that here the system is within the coexistence region), and thus the EC peak is cut off on the high$T$ side. For higher temperatures, above the coexistence region, the EC effect increases monotonously with the applied field strength. This is due to the high polarizability of the PE phase in this temperature and field region close to the phase transition, which leads to an increasingly large contribution from the configurational part and a broadening of the EC response towards higher temperatures. The characteristic peak shape with a sharp drop towards the low-T side and a field-dependent broad shoulder towards higher temperatures has also been observed experimentally in Ref. 12 ,

The off case is rather different from the on case, in particular for small fields. For the smallest applied field of $12 \mathrm{kV} / \mathrm{cm}$, the EC response is essentially negligible at all temperatures. In this case the random initialization with subsequent thermalization in the applied field has created a FE state for all temperatures below the ZFH transition temperature and a PE state above that, and no phase transition occurs under field removal (for the used $T$-sampling). For the next highest field of $20 \mathrm{kV} / \mathrm{cm}$ there is a sharp peak with $|\Delta T| \approx 2.6 \mathrm{~K}$ at $T_{\mathrm{i}}=304 \mathrm{~K}$, which is the only temperature for which a phase transition occurs under field removal for this initial field strength (cf. Fig. 4). This case is analogous to the case shown in Fig. 2(a) and already discussed above (note that for $20 \mathrm{kV} / \mathrm{cm}$ the width of the coexistence region at the C-T transition is only about $2 \mathrm{~K}$ and thus the initialization is not very crucial). For fields larger than $20 \mathrm{kV} / \mathrm{cm}$, the field strength approaches the critical field, above which there is no clear difference between $\mathrm{FE}$ and PE states. Instead, the system is highly polarizable and the EC peak broadens towards the higher temperature side for higher fields, similar to the on case. Thus, for fields above $E_{\mathrm{c}}$ the EC response becomes fully reversible, in the sense of Eq. (1), and the configurational (continuous) part of the EC effect becomes dominant over the part related to the FO transition entropy.

Towards the low- $\mathrm{T}$ side the EC peak exhibits a sharp drop, which is related to the stabilization of the FE phase for zero field. In the off case, this occurs when the system cools down during field removal and enters the coexistence region without undergoing a phase transition to the PE state. Since the configurational contribution to $\Delta T$ increases with increasing field strength, this low- $T$ side of the EC peak is shifted towards higher temperatures with increasing field. Note that this shift is overestimated in our simulations due to the overestimation of the unscaled $\Delta T$, as already discussed in Sec. II. This effect also leads to the non-monotonous behavior of $\Delta P$ at $T=304 \mathrm{~K}$ seen in Fig. 4(b), where for fields above $25 \mathrm{kV} / \mathrm{cm}$ the system remains in the FE state under field removal.

Summarizing the discussion related to Fig. 3, we find a reversible EC response for fields above the critical field strength for the FO PE-FE phase transition, whereas a strong difference (both in peak height and width) is observed between the on and off cases for small fields. This is in good agreement with experimental observations for BTO single crystals presented in Ref. 9 .

Finally, we examine the (ir-)reversibility of the EC response under field cycling near the maximum EC response close to the PE-FE (C-T) transition. Fig. 5 shows the time evolution of the system temperature and polarization as an external field of $E_{\text {app }}=12 \mathrm{kV} / \mathrm{cm}$ is applied and removed periodically during the course of the MD simulation starting from an initial temperature of $T_{i}=286 \mathrm{~K}$. Here, the system is initially in the PE-C phase, which transforms to the FE-T phase during the first application (ramp on) of the field. This results in a large EC temperature change, originating from the transitional contribution due to the FO phase transition (plus a small configurational part). However, on further field cycling the system always stays within the coexistence region, both with and without applied field, and thus it remains in the FE phase. Consequently, one observes only a much smaller EC response related to the configurational contribution. Thus, an irreversible heating of the system occurs during the first field field pulse, whereas a reversible but much smaller response appears during subsequent cycles. This is analogous to the magnetocaloric response observed at FO magneto-structural transitions, e.g., in Heusler alloys. 6 There, a large response is found only during the first field pulse, followed by a small but reversible response in subsequent cycles. 


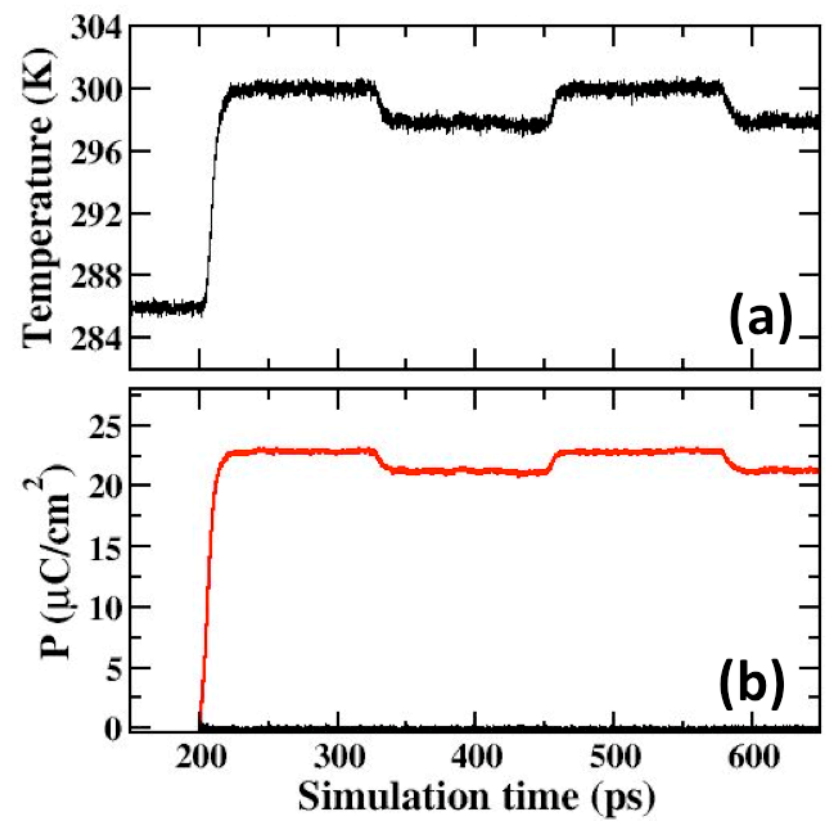

FIG. 5. Time evolution of the system temperature and polarization plotted during periodic cycling of a field of $E_{\text {app }}=$ $12 \mathrm{kV} / \mathrm{cm}$ for $T_{i}=286 \mathrm{~K}$. The system in initially in the PE-C phase. With the application of the field, there is a transition to the FE-T phase, leading to a large $\Delta T$ of $2.8 \mathrm{~K}$ (after rescaling). On subsequent removal of the field, the system remains in the FE-T phase and the EC response is much smaller.

\section{CONCLUSIONS AND OUTLOOK}

We have used $a b$ initio-based MD simulations for the prototypical ferroelectric material BTO to demonstrate several examples of irreversibility and the impact of thermal hysteresis on the EC response close to FO phase transitions. We found a large EC response, even for small applied electric fields, for cases where the system undergoes a FO phase transition. However, this can depend strongly on the thermal history of the sample and whether the EC effect is measured under field application or removal.

Although at the $\mathrm{C}-\mathrm{T}$ transition the coexistence region is narrow, a large difference between the EC responses observed under field application and removal, respectively, can occur at small fields. In particular, a large irreversible heating can arise during the first field application. This shows that it is important to measure the EC response for a well prepared initial state either under field removal or under field cycling in order to obtain reliable results for the reversible response.

At the T-O transition, the coexistence region is much broader, resulting in a nearly vanishing response in the FH case, since a small field is not sufficient to trigger the $\mathrm{FO}$ phase transition from the $\mathrm{O}$ to the $\mathrm{T}$ phase. Thus, a reversible cycling across the $\mathrm{T}-\mathrm{O}$ transition requires significantly larger field strengths.

Generally, for small fields the main contributions to the EC response results from the transition entropy when crossing the FO phase transition, whereas the configurational part is small but increases with increasing field strength. For the PE-FE (C-T) transition, the configurational part becomes dominant above the critical field strength $E_{\mathrm{c}}$, and at temperatures above the ZFH transition temperature. Therefore, hysteretic effects can be avoided by using sufficiently large applied fields, or by cycling the system above the ZFH transition temperature. In contrast, the configurational contribution to the EC effect is small within the FE phases near the T-O transition. We also note that, while a reversible cycling across the $\mathrm{T}-\mathrm{O}$ transition is in principle possible for very large fields, a partial cancellation between transitional and configurational parts can occur, since these two contributions can have opposite signs. 21

We note that the EC effect at the T-O transition shows several analogies to the well known giant magnetocaloric effect found at magneto-structural phase transitions, e.g. in Heusler alloys. ${ }^{7}$ There, a giant response is typically found for the first field pulse only, and the system stays within the coexistence range and shows a much smaller but reversible response under further field cycling. Furthermore, the structural (transitional) and magnetic (configurational) contributions to the magnetocaloric effect are opposite in sign, $\sqrt{29}$ similar to what can also occur a the T-O transition in BTO, as stated above. For the case of the magnetic materials, it has been shown that it is possible to bypass the hysteresis through an additional stimulus, such as, e.g., hydrostatic pressure. ${ }^{5}$ The same could also be possible for EC materials exhibiting strong hysteretic effects, due to the strong coupling of the $\mathrm{FE}$ degrees of freedom to pressure or strain.

Since our model is based on a defect-free homogeneous system, it allows to clearly isolate intrinsic and different extrinsic factors (such as, e.g., domains, defects, inhomogeneities, ...) determining hysteretic effects. In the present work we have focused on intrinsic properties, while the influence of domains, defects, and inhomogeneities can be incorporated in future studies. Our simulations therefore provide a good reference for comparison and analysis of future experimental investigations of hysteretic effect in EC materials.

\section{ACKNOWLEDGEMENT}

This work was supported by the Swiss National Science Foundation and the German Science Foundation under the priority program SPP 1599 ("Ferroic Cooling").

${ }^{1}$ J. Scott, "Electrocaloric materials," Ann. Rev. Mater. Res. 41, 229 (2011)

${ }^{2} \mathrm{M}$. Valant, "Electrocaloric materials for future solid-state refrigeration technologies," Prog. Mater. Sci. 57, 980 (2012).

${ }^{3}$ X. Moya, S. Kar-Narayan, and N. D. Mathur, "Caloric materials near ferrois phase transitions," Nature Mater. 13, 439 (2014).

${ }^{4}$ S. Fähler, U. K. Rössler, O. Kastner, J. Eckert, G. Eggeler, H. Emmerich, P. Entel, S. Müller, E. Quandt, and K. Albe, 
"Caloric effects in ferroic materials: New concepts for cooling," Adv. Eng. Mater. 14, 10 (2011).

${ }^{5}$ J. Liu, T. Gottschall, K. P. Skokov, J. D. Moore, and O. Gutfleisch, "Giant magnetocaloric effect driven by structural transitions," Nat. Mat. 11, 620 (2012).

${ }^{6}$ I. Titov, M. Acet, M. Farle, D. González-Alonso, L. Mañosa, A. Planes, and T. Krenke, "Hysteresis effects in the inverse magnetocaloric effect in martensitic Ni-Mn-In and Ni-Mn-Sn," J. Appl. Phys. 112, 073914 (2012).

${ }^{7}$ O. Gutfleisch, T. Gottschall, M. Fries, D. Benke, I. Radulov, K. P. Skokov, H. Wende, M. Gruner, M. Acet, P. Entel, and M. Farle, "Mastering hysteresis in magnetocaloric materials," Phil. Trans. R. Soc. A 374, 20150308 (2016).

${ }^{8}$ S. Kar-Narayan and N. D. Mathur, "Direct and indirect electrocaloric measurements using multilayer capacitors," J. Phys. D: Appl. Phys. 43, 032002 (2010).

${ }^{9}$ X. Moya, E. Stern-Taulats, S. Crossley, D. González-Alonso, S. Kar-Narayan, A. Planes, L. Mañosa, and N. D. Mathur, "Giant electrocaloric strength in single-crystal $\mathrm{BaTiO}_{3}$, , Adv. Mater. 25, 1360 (2013).

${ }^{10}$ N. Novak, R. Pirc, and Z. Kutnjak, "Impact of critical point on piezoelectric and electrocaloric response in barium titanate," Phys. Rev. B 87, 104102 (2013)

${ }^{11}$ G. Akcay, S. P. Alpay, J. V. Mantese, and G. A. Rossetti, "Magnitude of the intrinsic electrocaloric effect in ferroelectric perovskite thin films at high electric fields," Appl. Phys. Lett. 90, 252909 (2007)

${ }^{12}$ N. Novak, Z. Kutnjak, and R. Pirc, "High-resolution electrocaloric and heat capacity measurements in barium titanate," Euro. Phys. Lett. 103, 47001 (2013)

${ }^{13}$ S. Beckman, L. Wan, J. A. Barr, and T. Nishimatsu, "Effective Hamiltonian methods for predicting the electrocaloric behavior of $\mathrm{BaTiO}_{3}$," Mater. Lett. 89, 254 (2012)

${ }^{14}$ T. Nishimatsu, J. A. Barr, and S. P. Beckman, "Direct molecular dynamics simulation of electrocaloric effect in $\mathrm{BaTiO}_{3}$," J. Phys. Soc. of Jpn. 82, 114605 (2013)

${ }^{15} \mathrm{M}$. Marathe and C. Ederer, "Electrocaloric effect in $\mathrm{BaTiO}_{3}$ : A first-principles-based study on the effect of misfit strain," Appl. Phys. Lett. 104, 212902 (2014)

${ }^{10} \mathrm{~A}$. Grünebohm and T. Nishimatsu, "Influence of defects on ferroelectric and electrocaloric properties of batio 3 ," Phys. Rev. B 93, $134101(2016)$

${ }^{1 /}$ M. Marathe, A. Grünebohm, T. Nishimatsu, P. Entel, and C. Ederer, "First-principles-based calculation of the elec- trocaloric effect in $\mathrm{BaTiO}_{3}$ : A comparison of direct and indirect methods," Phys. Rev. B 93, 054110 (2016).

${ }^{18}$ M. E. Lines and A. M. Glass, Principles and Applications of Ferroelectrics and Related Materials (Oxford University Press, 1977).

${ }^{19}$ A. J. Bell, "Phenomenologically derived electric fieldtemperature phase diagrams and piezoelectric coefficients for single crystal barium titanate under fields along different axes," J. Appl. Phys. 89, 3907 (2001).

${ }^{20}$ Y. L. Li, L. E. Cross, and L. Q. Chen, "A phenomenological thermodynamic potential for $\mathrm{BaTiO}_{3}$ single crystals," J. Appl. Phys. 98, 064101 (2005)

${ }^{21}$ M. Marathe, D. Renggli, M. Sanlialp, M. O. Karabasov, V. V. Shvartsman, D. C. Lupascu, A. Grünebohm, and C. Ederer, "The electrocaloric effect in $\mathrm{BaTiO}_{3}$ at all three ferroelectric transitions: anisotropy and inverse caloric effects," Phys. Rev. B 96, $014102(2017)$

${ }^{22}$ Y. Bai, K. Ding, G.-P. Zheng, S.-Q. Shi, J.-L. Cao, and L. Qiao, "The electrocaloric effect around the orthorhombic- tetragonal first-order phase transition in $\mathrm{BaTiO}_{3}$," AIP Advances 2, 022162 (2012)

${ }^{23}$ W. Zhong, D. Vanderbilt, and K. M. Rabe, "Phase transitions in $\mathrm{BaTiO}_{3}$ from first principles," Phys. Rev. Lett. 73, 1861 (1994)

${ }^{24} \mathrm{~W}$. Zhong, D. Vanderbilt, and K. M. Rabe, "First-principles theory of ferroelectric phase transitions for perovskites: The case of $\mathrm{BaTiO}_{3}, "$ Phys. Rev. B 52, 6301 (1995)

${ }^{25}$ T. Nishimatsu, M. Iwamoto, Y. Kawazoe, and U. V. Waghmare, "First-principles accurate total energy surfaces for polar structural distortions of $\mathrm{BaTiO}_{3}, \mathrm{PbTiO}_{3}$, and $\mathrm{SrTiO}_{3}$ : Consequences for structural transition temperatures," Phys. Rev. B 82, $134106(2010)$

${ }^{26} \mathrm{~T}$. Nishimatsu, U. V. Waghmare, Y. Kawazoe, and D. Vanderbilt, "Fast molecular-dynamics simulation for ferroelectric thinfilm capacitors using a first-principles effective Hamiltonian," Phys. Rev. B 78, 104104 (2008)

${ }^{27}$ I. Ponomareva and S. Lisenkov, "Bridging the macroscopic and atomistic descriptions of the electrocaloric effect," Phys. Rev. Lett. 108, 167604 (2012)

${ }^{28}$ We note that for a ZFC starting configuration, the steep rise on the low- $T$ side of the EC peak occurs exactly at the ZFC transition temperature.

${ }^{29}$ T. Gottschall, K. P. Skokov, D. Benke, M. E. Gruner, and O. Gutfleisch, "Contradictory role of the magnetic contribution in inverse magnetocaloric heusler materials," Phys. Rev. B 93, $184431(2016)$ 